\title{
Analysis of Brainwave Characteristics for Playing Heterogeneous Computer Games
}

\author{
Fu-Chien Kao, Han-Chien Hsieh, and Wei-Te Li \\ Computer Science \& Information Engineering, Dayeh University, Taiwan, ROC \\ \{fuchien, R9906020,R9906031\} @mail.dyu.edu.tw
}

\begin{abstract}
The traditional E-learning often offers the online examination to assess the learning effect of a student after completion of the online learning. Basically, this traditional learning assessment mechanism is a passive and negative assessment mechanism, which cannot provide an real-time learning warning mechanism for teachers or students to find out problems as early as possible (including such learning conditions as "absence of mind" resulting from poor learning stage or physical or psychological factor), and the post-assessment mechanism also cannot assess the learning effectiveness provided by the online learning system. This research paper attempts to acquire the electroencephalogram to analyze the characteristic frequency band of the brainwave related to learning and formulate the learning energy index (LEI) for the learner at the time when the learner is reasoning logically via the brain-wave detector based on the cognitive neuroscience. With the established LEI, the physical and psychological conditions of an online leaner can be provided instantly for teachers for assessment. Given that the learning system is integrated into the brainwave analytic sensing component, the system not only can provide learners an instant learning warming mechanism, but also help teachers and learning partners to further understand the causes of learning disorder of learners, and can also provide relevant learning members with timely care and encouragement. From the analysis of the experimental results, it is to prove that the game-based learning has not only the energy distribution of the characteristic frequency band the same as that by using professional textbooks, but also the way of game design can enhance the LEI of learners more in the aspect of training logical reasoning.
\end{abstract}

Keywords: Cognitive neuroscience, Learning energy index, Electroencephalogram, Game-based learning. 